\title{
The Role of Comorbidity on Retention in HIV Care
}

\author{
Shiraze M. Bulsara ${ }^{1,2}$ (1) $\cdot$ Milton L. Wainberg ${ }^{3} \cdot$ Kris Rogers $^{1} \cdot$ John McAloon ${ }^{1} \cdot$ Rachel Grove $^{1}$. \\ Toby R. O. Newton-John ${ }^{1}$
}

Published online: 5 August 2020

(c) Springer Science+Business Media, LLC, part of Springer Nature 2020

\begin{abstract}
Retention is a central component of the Cascade, facilitating monitoring of comorbidity. Country-specific definitions differ and may suit stable and functioning clients, while not appropriately classifying complex clinical presentations characterized by comorbidity. A retrospective file review of 363 people living with HIV attending a Sydney HIV clinic was conducted. Retention was compared with Australian (attendance once/12-months) and World Health Organization (attendance 'appropriate to need') recommendations to identify those attending according to the Australian definition, but not clinician recommendations (AUnotWHO). Multivariable logistic regression analyses determined the impact of age/sex and clinician-assessed comorbidity on retention. Most (97\%) participants were considered retained according to the Australian definition, but only $56.7 \%$ according to clinician recommendations. Those with psychosocial comorbidity alone were less likely to be in the AUnotWHO group $(O R 0.51,95 \% \mathrm{CI} 0.27-0.96, p=0.04)$. The interaction of physical and psychosocial comorbidity was predictive of poor retention (Wald test: $\chi^{2}=6.39$, OR 2.39 [95\% CI 1.15-4.97], $p=0.01$ ), suggesting a syndemic relationship.
\end{abstract}

The lead author is a 2019 recipient of the NSW Health Education and Training Institute (HETI) Mental Health Research Award. The Albion Centre is a NSW Health entity, and WHO Collaborating Centre for capacity building on HIV and STI Care, treatment and support.

Shiraze M. Bulsara

shiraze.bulsara@health.nsw.gov.au

Milton L. Wainberg

mlw35@cumc.columbia.edu

Kris Rogers

kris.rogers@uts.edu.au

John McAloon

john.mcaloon@uts.edu.au

Rachel Grove

rachel.grove@uts.edu.au

Toby R. O. Newton-John

toby.newton-john@uts.edu.au

1 Clinical Psychology, Graduate School of Health, University of Technology Sydney (UTS), Sydney, NSW, Australia

2 The Albion Centre, 150 Albion Street, Surry Hills, NSW 2010, Australia

3 Department of Psychiatry, Columbia University, New York, NY, USA

\section{Introduction}

The changing nature of HIV from an acute to a chronic illness due to biomedical advances has heralded a new era of HIV management. HIV service providers are increasingly recognizing the importance of regular monitoring of psychosocial, as well as medical, comorbidity in the context of long term HIV management, including viral suppression [1]. Despite this, outcomes and metrics for treatment success remain solely biomedical, which is considered problematic by many [2]. One aspect of the HIV Treatment Cascade (Cascade), by which region-specific responses to the HIV epidemic are evaluated and relevant resources/funding allocated, is retention in care, which accounts for regular monitoring of people living with HIV.

Definitions of retention vary within and between regions. Current definitions in some developed countries might be considered overly specific. In Australia and the U.K, people living with HIV are considered retained in care if they attend at least once in a 12-month period, with viral load (VL) testing often used as a proxy $[3,4]$. In the U.S, people living with HIV are considered retained if they attend at least two medical appointments in 12 months, at least 90 days apart [5]. In Australia specifically, where VL is a single outcome measure, this might suit relatively stable, functioning clients, 
but may neglect to adequately identify those with more complex medical and/or psychosocial needs.

The World Health Organization (WHO) revised their definition of retention in care to "attendance appropriate to [the] need" [6, p.26]. Under this definition, 'need' could be determined by the schedule of appointments indicated by the HIV specialist, who can assess the impact of all medical and/ or psychosocial factors and any need for intervention and/ or referrals. Definitions of retention, which guide resource allocation, are relied upon to provide a valid representation of healthcare needs and delivery within regions. If they are too narrow and do not capture variation across the spectrum of comorbidity, the validity of the data is questionable and there is potential to negatively bias client care and resource allocation. Therefore, this definition in the context of specialist assessment may represent a more clinically meaningful and valid assessment of retention.

Clinical presentations rarely present in isolation and most often present as a complex interplay of factors, which increases the resources required for effective treatment [1], and is unlikely to require the same frequency/schedule of visits as someone whose HIV-infection and associated comorbidity is relatively well-managed. Understanding the profile of comorbidity which impacts retention rates is therefore important for longevity of care in people living with HIV. A syndemic lens considers the interaction of various factors which, when taken together, can increase adverse health outcomes or health disparities [7]. Many studies have considered the impact of syndemics on Cascade outcomes, including retention in care [8-10]. In the context of an ageing cohort of people living with HIV, understanding this comorbidity, or potential syndemic, is necessary to target interventions and appropriately allocate resources.

In determining an individual's schedule of visits, HIV medical specialists consider a range of factors. The nature and chronicity of the difficulties identified, as well as the degree and coordination of care required, are central components in the primary clinician's decision-making regarding treatment, referrals, and appointment schedule [1]. Historically, such assessments have been unstandardized, leaving the validity of the data questionable. Three studies to our knowledge have attempted to standardize the assessment; Grant and colleagues recognized the important role of clinician-identified comorbidity/complexity, and sought to quantify this with a U.S. population [1]. Likewise, Howarth and colleagues recognized the importance of other factors such as treatment and health status in reporting retention, and sought to incorporate these factors into their risk prediction tool $[11,12]$. These authors noted that, although psychosocial comorbidities were often central in determining the schedule and frequency of follow-up visits, this data is not consistently captured in medical records and therefore could not be included in their algorithm [12]. A third study developed a brief risk-prediction screening tool of clinicianassessed physical and psychosocial comorbidity and clinical complexity for people living with HIV in Sydney, Australia [13]. Such research highlights a way to capture the characteristics of those at risk of sub-optimal appointment adherence in a standardized manner.

The present study sought to compare attendance to medical HIV appointments against local (Australian) region-specific estimates of retention, as well as the WHO guidelines. Like other retrospective studies, only routinely collected variables were assessed; however, this study differs from previous retrospective research in this area in that it provides an assessment of medical and/or psychosocial comorbidity. It is expected that people living with HIV with clinicianidentified comorbidity and complex clinical presentations will be less likely to regularly attend appointments during the study period. Furthermore, some discrepancy between region-specific estimates and individualized schedules of retention is expected.

\section{Methods}

\section{Participants}

A clinical file audit of people living with HIV who attended The Albion Centre (Albion), a publicly funded interdisciplinary HIV service in metropolitan Sydney, Australia, was conducted. A number of private and public HIV services are located close-by; unfortunately, no centralized database capturing attendance exists, making it impossible to identify whether clients had attended at another local service. As a result, only attendance to Albion was captured. Albion typically supports clients from a range of ethnic and cultural backgrounds, from all over Sydney. Many clients present with a range of complex comorbid presentations requiring input from a number of clinical services within the clinic. The medical records of those who attended the clinic for medical review between 01/02/17 and 30/04/17 were monitored for a 13-month period. Exclusion criteria are detailed in Fig. 1. The data extracted related to the next appointment recommended by the Attending Medical Officer (AMO), determined by individual clinical assessment of holistic care needs. Ethics approval was obtained through the South East Sydney Local Health District (Ref: LNR/16/POWH/530) and the University of Technology (Ref: 2015000482-30) HREC Committees.

\section{Outcome Variable}

Retention in care was the primary outcome variable of interest, measured against two key definitions: 
Fig. 1 Summary of 'occasions of service' with inclusion and exclusion criteria

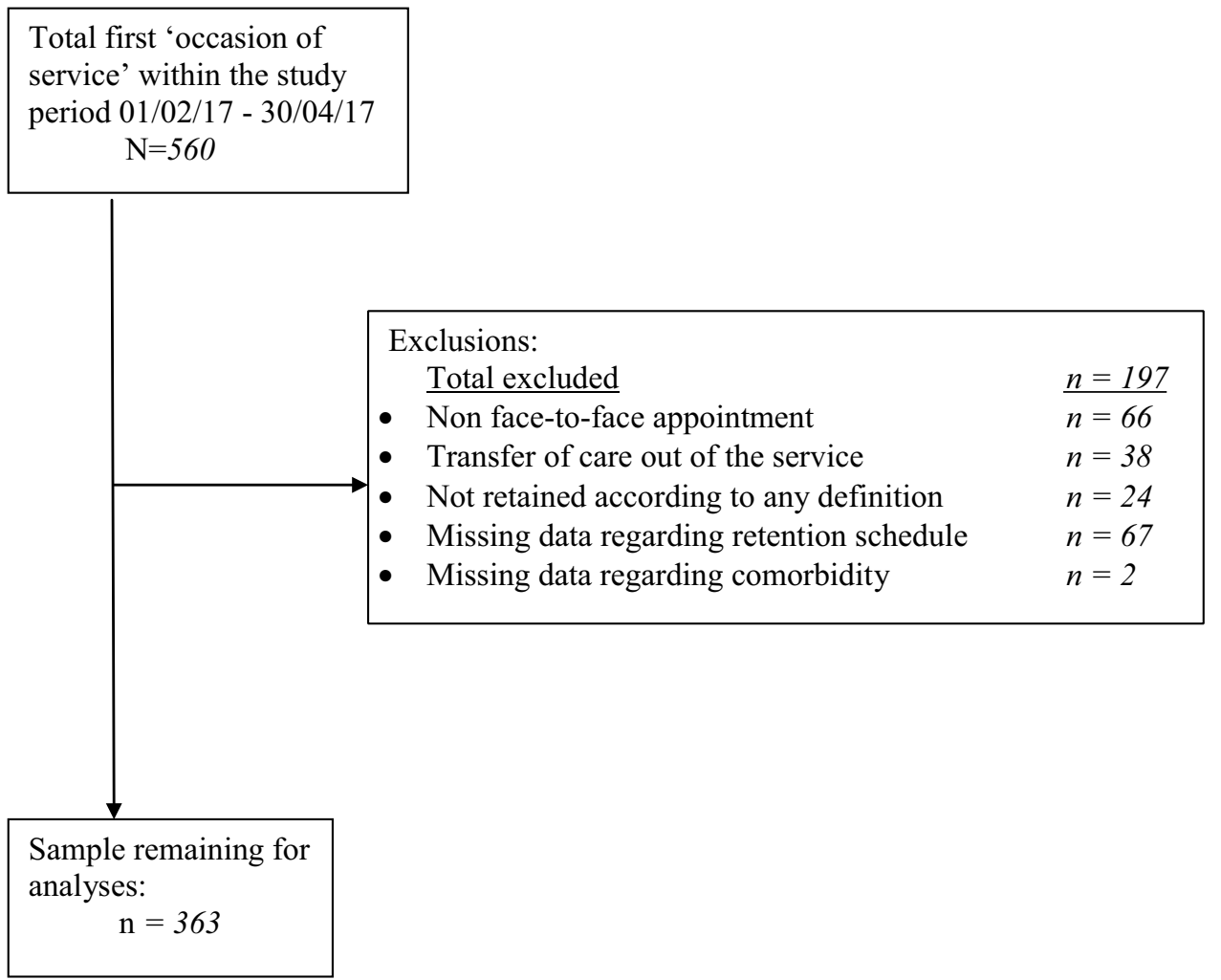

\section{Australia- “having had a viral load or CD4+ cell count} in the past year" [3].

WHO_Attendance for medical review "appropriate to the need" [6]. For the present study, this was conceptualized as adherence to an individualized schedule of visits over the 13-month period, determined by an HIV specialist.

Multiple metrics of retention exist in the literature including missed clinic visits, gaps in care, and appointment adherence [14]; in the context of the WHO definition, appointment adherence $\left(P_{\text {attended }}\right)$ was considered the most appropriate measure. This details the proportion of visits attended on time relative to the total number recommended:

$P_{\text {attended }}=\frac{\# \text { visits attended on time }}{\# \text { total recommended visits }}$

There is no clear precedent in the literature to operationalize (a) the timeframe within which 'on time' attendance would be considered optimal, and (b) the proportion of visits which needed to be included within that timeframe; as such, these parameters had to be defined here. It is worth noting that an SMS appointment reminder system is in place, which reminds clients of their appointment the day prior. In consultation with HIV specialists at Albion, 'on time' attendance, for medical HIV appointments only, was defined as within one-month/30 days of the scheduled appointment. Attendance to other nursing and/or allied health appointments were not included as these are not consistent with current definitions of retention in care. A conservative target proportion of attendance at $75 \%$ of scheduled visits was chosen for the present study. A binary variable was created according to this definition, in which a target group (AUnotWHO) was categorized as those considered retained in care according to the Australian definition, but not according to the WHO definition (i.e. who did not attend at least $75 \%$ of visits within one-month/30 days of the schedule determined by their AMO over the 13-month study period). The study period was set at 12-months; however, as the definition of attendance was attending within one month/30 days of the scheduled appointment, the actual study period was 13-months.

\section{Client Characteristics and Predictor Variables}

Given the retrospective design of the study, only routinely collected variables were reviewed, including age and sex. All clients attending Albion for medical HIV care had been assessed by their treating AMO for medical and/or psychosocial comorbid presentations, using the Clinical Complexity Rating Scale for HIV [CCRS-HIV; [13]]. This 8-item tool has been validated to screen for complex medical and/ or psychosocial comorbidity. There are four psychosocial variables (social isolation; problematic crystal methamphetamine [CMA] use; financial instability; mental illness and/or other problematic substance use), and four physical health (cognitive/neurological impairment; polypharmacy; current Hepatitis C [HCV] and/or cancer; other physical health comorbidity) variables. It is a clinician-reported 
screening tool of functional impairment as a result of factors commonly impacting people living with HIV (Table 1). Scores are weighted, acknowledging that variables do not all have the same degree of impact on functioning. During validation, the screening tool was shown to accurately predict $85 \%$ of complex clients, with a sensitivity of $80 \%$ and specificity of $91 \%$ [13]. Examples of decision-making when scoring the tool include "Does financial instability impact the person's current functioning?", or "Does the presence of other physical health comorbidity impact the person's current functioning?". In the validation study, those with a total score of 0-29 were considered in the 'low' complexity range, those with a total score of 30-39 in the 'medium' complexity range, and those with a score of 40 or greater in the 'high' complexity range [13]. Scores were then summed in both domains. Domain scores rather than categories were used in the present analyses. Those who scored 'Yes' to a physical variable were given a ' 1 ' on the Physical Health Complexity Domain, and similarly any endorsement of a psychosocial variable resulted in a score on the Psychosocial Complexity Domain.

\section{Analyses}

The data were examined to characterize the "AUnotWHO group'. Retention rates according to each definition and client characteristics were summarized, with means and standard deviations for continuous measures and counts and proportions (of total sample) for binary variables calculated (Table 2). Given the focus on comorbidity, we used multivariable logistic regression analyses to assess the impact of client characteristics (age and sex) and clinician-assessed Physical Health (PH) and/or Psychosocial (PSY) complexity on the probability of membership to the AUnotWHO group. With the risk of quasi-separation from the small absolute number of outcomes, all analyses were conducted using a penalized regression model with Firth correction [15].

To assess for the presence of a syndemic, interactions between the PH and PSY complexity domains were

Table 1 Summary of variables and associated complexity domains in the CCRS-HIV

\begin{tabular}{ll}
\hline Complexity domain & Variable \\
\hline Psychosocial (PSY) & Financial instability \\
& Social isolation \\
& Problematic Crystal Methampheta- \\
mine (CMA) use \\
Mental illness \&/or other problem- \\
atic substance use \\
Cognitive/neurological impairment \\
Polypharmacy \\
Physical health (PH) & Other physical health comorbidity \\
\hline
\end{tabular}

included, consistent with the model outlined in Tsai \& Venkataramani [16]. A fully saturated logistic regression model to capture interactions was assessed:

$Y=\alpha+\beta_{1} X_{1}+\beta_{2} X_{2}+\beta_{3} X_{1} X_{2}$

Exploratory analyses were then conducted between all first order interactions of the complexity variables.

All models showed acceptable levels of goodness-of-fit according to the Hosmer-Lemeshow statistic [17]. Linearity of age (years) was checked visually by plotting the logit values against age. Inclusion in the AUnotWHO group was determined by odds ratios (OR), such that an OR $>1$ indicated increased odds of inclusion, and $\mathrm{OR}<1$ showed decreased odds of inclusion. Analyses were conducted using SPSS v.25 (SPSS v.25, Armonk, NY, USA) and SAS 9.4 (SAS/STAT 15.0, Cary, NC, USA).

\section{Results}

\section{Descriptive Statistics}

\section{Inclusion and Exclusion of Cases}

A total of 560 'first occasions of service' were documented within the study period. After exclusions a total of $n=363$ remained. Exclusion criteria were determined to capture those who attended, in person, for the first medical HIV visit of the study period. As such, non face-to-face appointments were excluded. Those who were not retained according to either the Australian or the WHO definition were also excluded from the analyses as the focus of the present study was to identify those who were retained according to the Australian definition, but were not attending 'appropriate to need'. In addition, those who had transferred out of the service during the study period were excluded, as they were no longer accessing the service for their medical HIV care. Finally, those with significant missing data regarding their clinician-identified comorbidity or their clinician-determined attendance schedules were excluded, as this information was necessary for the analyses. Figure 1 summarizes inclusion and exclusion criteria. To ensure each client was only captured once (i.e. no repeat visits), subsequent visits within the study period were excluded.

\section{Demographics}

The mean age of participants was 47.36 years $(\mathrm{sd}=12.42$; range 19-80 years; Table 2). The sample predominantly consisted of males $(91.2 \%)$, consistent with the profile of the HIV-epidemic in Australia [3] and Albion's client-base. 
Table 2 Summary of retention in care (outcome) and covariate variables and descriptive statistics

\begin{tabular}{|c|c|c|c|c|c|c|}
\hline \multirow[t]{2}{*}{ Variable category } & \multirow[t]{2}{*}{ Category } & \multirow[t]{2}{*}{ Variables } & \multicolumn{2}{|l|}{ Whole sample } & \multicolumn{2}{|c|}{ AUnotWHO group } \\
\hline & & & $\begin{array}{l}\text { Mean (SD)/ } \\
\text { Median (p25- } \\
\text { p75) }\end{array}$ & $\begin{array}{l}\text { Number of } \\
\text { participants } \\
(\%)\end{array}$ & $\begin{array}{l}\text { Mean }(\mathrm{SD}) / \\
\text { median }(\mathrm{p} 25- \\
\mathrm{p} 75)\end{array}$ & $\begin{array}{l}\text { Number of } \\
\text { participants } \\
(\%)\end{array}$ \\
\hline \multirow[t]{2}{*}{ Outcome } & \multirow[t]{2}{*}{ Retained (AU guidelines) } & No & - & $11(3.0)$ & - & 0 \\
\hline & & Yes & - & $352(97.0)$ & - & $149(100)$ \\
\hline \multirow[t]{2}{*}{ Outcome } & \multirow{2}{*}{$\begin{array}{l}\text { Retained (AU + WHO } \\
\text { guidelines) }\end{array}$} & No (AUnotWHO Group) & - & $149(41.0)$ & - & - \\
\hline & & Yes & - & $214(59.0)$ & - & - \\
\hline \multirow[t]{4}{*}{ Covariate } & \multirow[t]{4}{*}{ Demographics } & Age (years) & $\begin{array}{l}47.36(12.42) / \\
48(37-56)\end{array}$ & - & $\begin{array}{l}46.55(12.9) / \\
47(37-55)\end{array}$ & - \\
\hline & & Sex & & & & \\
\hline & & Male & - & $331(91.2)$ & - & $133(88.7)$ \\
\hline & & Female & - & $32(8.8)$ & - & $17(11.3)$ \\
\hline \multirow[t]{4}{*}{ Covariate } & Psychosocial (PSY) & Financial instability (Yes) & - & $50(13.8)$ & - & $22(14.8)$ \\
\hline & \multirow[t]{3}{*}{ Complexity domain } & $\begin{array}{l}\text { Dysfunctional CMA use } \\
\text { (Yes) }\end{array}$ & - & $25(6.9)$ & - & $12(8.1)$ \\
\hline & & $\begin{array}{l}\text { Mental illness \&/or other } \\
\text { problematic substance use } \\
\text { (Yes) }\end{array}$ & - & $101(27.8)$ & - & $41(27.5)$ \\
\hline & & Social Isolation (Yes) & - & $65(17.9)$ & - & $27(18.1)$ \\
\hline \multirow[t]{4}{*}{ Covariate } & Physical health $(\mathrm{PH})$ & $\begin{array}{l}\text { Cognitive/neurological } \\
\text { impairment (Yes) }\end{array}$ & - & $24(6.5)$ & - & $9(6.0)$ \\
\hline & \multirow[t]{3}{*}{ Complexity domain } & Polypharmacy (Yes) & - & $46(12.7)$ & - & $18(12.1)$ \\
\hline & & HCV \&/or Cancer (Yes) & - & $18(5.0)$ & - & $6(4.0)$ \\
\hline & & $\begin{array}{l}\text { Other physical health comor- } \\
\text { bidity (Yes) }\end{array}$ & - & $119(32.8)$ & - & $49(32.9)$ \\
\hline \multirow[t]{4}{*}{ Covariate } & \multirow{4}{*}{$\begin{array}{l}\text { PH and PSY Complexity } \\
\text { Domains }\end{array}$} & Neither PH nor PSY & - & $154(42.4)$ & - & $67(45.0)$ \\
\hline & & PH complexity domain only & - & $70(19.3)$ & - & $28(18.8)$ \\
\hline & & $\begin{array}{l}\text { PSY complexity domain } \\
\text { only }\end{array}$ & - & $65(17.9)$ & - & $19(12.8)$ \\
\hline & & Both PH and PSY & - & $74(20.4)$ & - & $35(23.5)$ \\
\hline
\end{tabular}

\section{Retention in Care}

Most clients were retained according to the Australian definition (97\%); however, considerably fewer (56.7\%) were found to be reliably attending appointments as recommended by their AMO, consistent with WHO guidelines. The AUnotWHO group was $n=149(41.0 \%)$, indicating that $59.0 \%$ of the sample attended appointments consistent with both definitions. These data are summarized in Table 2.

\section{Characteristics of the Whole Sample}

The profile and frequency of comorbidity identified by clinicians using the CCRS-HIV [13] are presented in Table 2. The majority $(71.6 \%)$ of the sample were classified in the 'low' complexity range (with $42.4 \%$ not scoring on any variable), $7.7 \%$ in the 'medium' range, and a further $20.7 \%$ in the 'high' range. The majority of the sample (97\%) had an undetectable viral load (UDVL) during the study period.

\section{Characteristics of the AUnotWHO Group}

The AUnotWHO group $(\mathrm{n}=149)$ comprized 132 males (88.6\%) and 17 females (11.4\%). Fifteen (10.1\%) had a detectable VL in the AUnotWHO group; of all participants with a detectable VL in the total sample, $62.5 \%$ of them were in the AUnotWHO group. Within the AUnotWHO group, $71.1 \%$ were assessed according to the CCRS-HIV to be in the 'low' complexity range, indicating that most participants in this group did not have significant clinicianidentified PSY \&/or PH comorbidity. A total of $6.7 \%$ were in the 'medium' complexity range, and $22.1 \%$ in the 'high' complexity range.

\section{Comorbidity Predictors of Retention}

Table 3 outlines the results of binary logistic regression models. Goodness-of-fit was assessed using the Hosmer-Lemeshow goodness-of-fit test [17]. The first 
Table 3 Multivariable binary logistic regression results for 1 ) Age and Sex, 2) Comorbidities (with their interaction) and Age and Sex

\begin{tabular}{|c|c|c|c|c|c|}
\hline \multirow[t]{2}{*}{ Analyses } & \multirow[t]{2}{*}{ Variables } & \multirow{2}{*}{$\begin{array}{l}\text { Odds Ratio (OR) of AUnot- } \\
\text { WHO group membership }\end{array}$} & \multicolumn{2}{|c|}{$95 \% \mathrm{CI}$ for OR } & \multirow[t]{2}{*}{$\mathrm{p}$} \\
\hline & & & Lower & Upper & \\
\hline \multirow[t]{3}{*}{ Model 1 (age and sex) } & Age (years) & 0.99 & 0.98 & 1.01 & 0.56 \\
\hline & Sex (Male) & REF & & & \\
\hline & Sex (Female) & 1.70 & 0.79 & 3.66 & 0.17 \\
\hline \multirow{7}{*}{$\begin{array}{l}\text { Model } 2 \text { (comorbidity } \\
\text { with age and sex) }\end{array}$} & Age (years) & 0.99 & 0.97 & 1.01 & 0.23 \\
\hline & Sex (Male) & REF & & & \\
\hline & Sex (Female) & 1.77 & 0.84 & 3.74 & 0.13 \\
\hline & $\mathrm{PH}=0, \mathrm{PSY}=0$ & REF & & & \\
\hline & $\mathrm{PH}=1, \mathrm{PSY}=0$ & 0.98 & 0.54 & 1.78 & 0.93 \\
\hline & $\mathrm{PH}=0, \mathrm{PSY}=1$ & 0.51 & 0.27 & 0.96 & 0.04 \\
\hline & $\mathrm{PH}=1, \mathrm{PSY}=1$ & 1.22 & 0.68 & 2.19 & 0.50 \\
\hline
\end{tabular}

model, including only covariates, showed no significant impact of age, sex, or VL on retention. The second model estimated the role of PH and PSY comorbidity, as well as their interaction, on retention (Table 3). Both models were considered a reasonable fit $(p=0.86$ and $p=0.66$ respectively). A combined variable using dummy codes was created using neither PH nor PSY as the reference category, indicating those with PSY comorbid factors had significantly lower odds $(0.51,95 \%$ CI $0.27-0.96$, $p=0.04$ ) of AUnotWHO group membership. Given this result, the dummy variable was re-coded to estimate the additional impact of PH comorbidity on PSY, using PSY alone as the new reference category. Compared to PSY only, no comorbidity was associated with higher odds of being in the AUnotWHO group (OR 1.96, 95\% CI 1.04-3.69, $p=0.04$ ), and clients with both PH and PSY comorbidity had greater odds of being in the AUnotWHO group (OR 2.39, 95\% CI 1.15-4.97, $p=0.02$ ). These results suggest the presence of a syndemic of $\mathrm{PH}$ and PSY complexity factors [16].

\section{Exploratory Analyses}

To investigate the relationships between specific comorbidity variables in the context of the identified PH*PSY interactional effect on retention, exploratory analyses were conducted between first order variables. These results are presented in Table 4. All combinations $(n=28)$ were explored, and we identified an interaction between problematic CMA use and social isolation (Wald test: $\chi^{2}=6.39,95 \%$ CI 1.18-9.29, $p=0.01$ ). This showed that those who were considered by their clinician to be socially isolated (OR 1.48, 95\% CI 0.92-2.4) or have problematic CMA use (OR 1.19, 95\% CI 0.74-1.93) alone were not more likely to be in the target group, but those with both social isolation and problematic CMA use were more likely (OR 3.3, 95\% CI 1.17-9.28) than those with neither to be in the target group.
At this point, we reached the limits of the data and could not explore further (e.g. $3 \times 3$ ) interactions.

\section{Discussion}

The present study is the first, to our knowledge, to compare estimates of retention based on region-specific definitions to those determined by the WHO. As anticipated, there was a discrepancy between the two estimates for the same cohort, with 97\% considered retained according to Australian definitions, but a AUnotWHO group of $41.0 \%$ retained according to Australian guidelines yet not adhering to a schedule of visits determined by an HIV specialist.

Characteristics of the AUnotWHO group indicate the majority $(71.1 \%)$ scored in the low range on the CCRSHIV, and $28.8 \%$ of the cohort in the medium-high complexity bracket. This indicates that, for most individuals, their physical and psychosocial comorbidity as determined by the CCRS-HIV did not necessarily impact their retention. However, this does not discount the need to monitor those with complex care needs who are likely to be more vulnerable.

Contrary to our expectations, we found that those with identified psychosocial comorbidity were less likely to be in the AUnotWHO group. However, it is possible that in this sample, those with identified psychosocial comorbid factors were more likely to be engaged with the psychology unit within the service. Future research in this area should seek to quantify this assertion. Increased points of connection with a service may, in fact, enhance retention [18, 19]. Further, those who are socially isolated, one factor which comprised the psychosocial domain, could be accessing services more frequently to address their unmet social needs [20]. Likewise, health-related worry can lead this group to over-access services [21].

Compared to this cohort with psychosocial comorbidity alone, when physical health issues were also present a significant interaction of the two variables increased the odds 
Table 4 Exact Wald tests with Firth Bias correction for exploratory interaction analyses

\begin{tabular}{|c|c|c|}
\hline Interaction & Test statistic $\left(\chi^{2}\right)$ & $p$ \\
\hline Financial instability*Problematic CMA use & 2.36 & 0.12 \\
\hline Financial Instability* Mental illness \&/or other problematic substance use & 1.17 & 0.28 \\
\hline Financial Instability*Isolation & 0.58 & 0.45 \\
\hline Financial Instability*Cognitive/neurological impairment & 0.54 & 0.46 \\
\hline Financial Instability*Polypharmacy & 0.22 & 0.64 \\
\hline Financial Instability*HCV \&/or Cancer & 0.03 & 0.86 \\
\hline Financial Instability*Other physical health comorbidity & 0.06 & 0.81 \\
\hline Problematic CMA use* Mental illness \&/or other problematic substance use & 1.00 & 0.32 \\
\hline Problematic CMA use*Isolation & 6.39 & 0.01 \\
\hline Problematic CMA use*Cognitive/neurological impairment & 0.20 & 0.66 \\
\hline Problematic CMA use*Polypharmacy & $<0.01$ & 1.00 \\
\hline Problematic CMA use*HCV \&/or Cancer & $<0.01$ & 0.95 \\
\hline Problematic CMA use*Other physical health comorbidity & 0.01 & 0.92 \\
\hline Mental illness \&/or other problematic substance use*Isolation & 0.59 & 0.44 \\
\hline Mental illness \&/or other problematic substance use*Cognitive/neurological impairment & 0.05 & 0.82 \\
\hline Mental illness \&/or other problematic substance use*Polypharmacy & 0.09 & 0.76 \\
\hline Mental illness \&/or other problematic substance use*HCV \&/or Cancer & 1.59 & 0.21 \\
\hline Mental illness \&/or other problematic substance use* Other physical health comorbidity & 0.22 & 0.64 \\
\hline Isolation*Cognitive/neurological impairment & 0.42 & 0.52 \\
\hline Isolation*Polypharmacy & 0.19 & 0.66 \\
\hline Isolation*HCV \&/or Cancer & 0.48 & 0.49 \\
\hline Isolation* Other physical health comorbidity & 0.81 & 0.37 \\
\hline Cognitive/neurological impairment*Polypharmacy & $<0.01$ & 0.98 \\
\hline Cognitive/neurological impairment*HCV \&/or Cancer & 0.03 & 0.86 \\
\hline Cognitive/neurological impairment*Other physical health comorbidity & 0.49 & 0.48 \\
\hline Polypharmacy*HCV \&/or Cancer & 0.31 & 0.58 \\
\hline Polypharmacy*Other physical health comorbidity & 2.34 & 0.13 \\
\hline HCV \&/or Cancer*Other physical health comorbidity & 2.70 & 0.10 \\
\hline
\end{tabular}

Fig. 2 A syndemic model of the synergistic effect of combined PH and PSY complexity domains on retention in care, as outlined in [22]

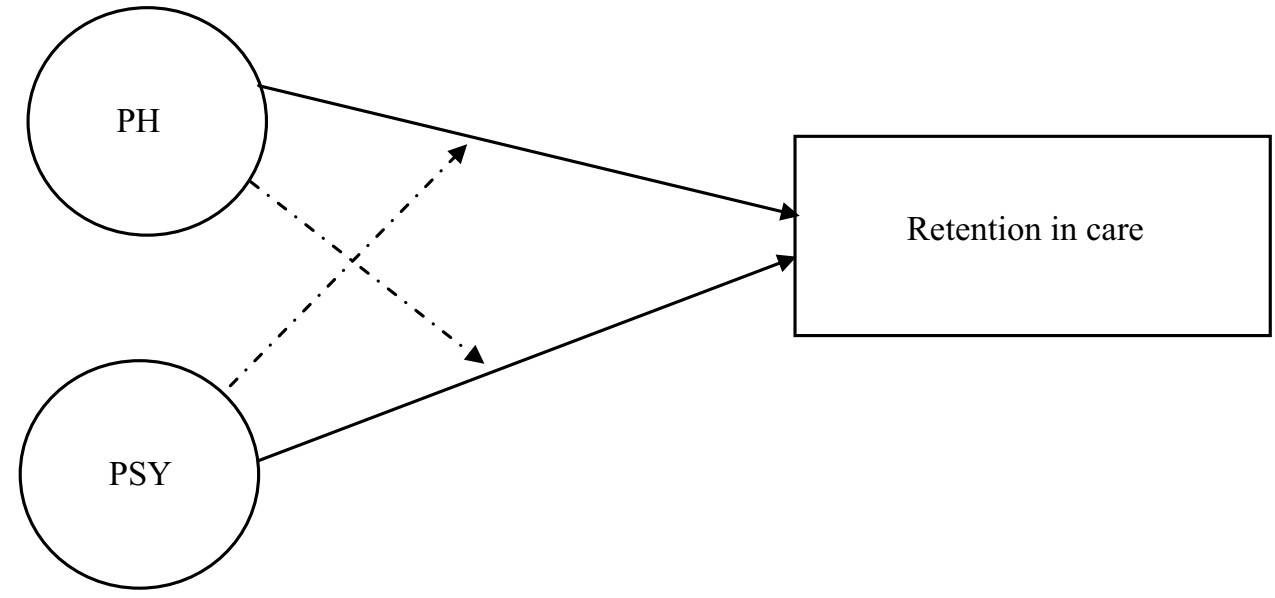

PH, Physical Health factors; PSY, Psychosocial factors of being in the AUnotWHO group. This result is consistent with a syndemic approach to disease interaction [16], suggesting a synergistic effect of physical and psychosocial variables in this cohort. Figure 2 accounts for the potential relationship between these factors, consistent with the model suggested by Tsai et al. [22]. 
Interestingly, those with no clinician-identified comorbidity had an increased odds of being in the AUnotWHO group compared the psychosocial-alone group. It may be that these clients 'feel well' and therefore do not prioritize their appointments [19]. This could also account for the discrepancy in retention estimates, with clients potentially not agreeing with the schedule determined by their Doctor. In the present study, it is also possible that clients received antiretroviral therapy (ART) prescriptions between appointments, which could potentially decrease the need to attend appointments on schedule. It was beyond the scope of the present study to assess for this potentially confounding factor, however future research in this field should seek to address this.

Exploratory analyses identified a significant interaction between problematic CMA use and social isolation. Other studies have found similar results; a recent Australian study found that CMA use was associated with higher rates of depressive and anxious symptoms, and poorer social engagement [23], and a Danish study conducted in a comparable setting to that of this study, noted that intravenous drug use was associated with poor retention in care [24]. The combination, or synergistic interaction, of these factors therefore could limit a person's capacity for self-care, including attending healthcare appointments. As the limits of the data were reached, further (e.g. $3 \times 3$ ) interactions could not be explored; we were therefore unable to identify the specific component of the physical complexity domain which contributed to the significant interaction, suggesting a gap in this research. While generalizability of this finding is low given low cell numbers, the result is a promising foundation for future research to specifically assess this potential syndemic and its role on retention.

The results of this study point to multiple factors (comorbidity) influencing retention, especially the identified syndemic of physical and psychosocial complexity factors. Despite simplifications in ART regimes and advances in HIV medical management, retention in care remains an important component of the Cascade, as it allows experienced clinicians to monitor all factors from a biopsychosocial, holistic framework [25]. This is impossible to achieve through annual phlebotomy tests alone. Including more holistic markers of treatment success (e.g. quality of life) would potentially capture a more comprehensive biopsychosocial framework. Current definitions of retention most suit those who are relatively stable and functioning well, and potentially redirect necessary funding and resources away from services who manage complex clinical presentations.

While the results of the present study suggest comorbid presentations might not detrimentally impact functioning at this point, they do require attention. Proactive management of an ageing cohort involves identifying risk factors before they detrimentally impact health outcomes. Physical, as well as psychosocial, comorbidity has the potential to impact not only functioning, but also biomedical outcomes such as VL [26]. Effectively managing comorbidity, facilitated by retention, requires individual monitoring, assessment, and schedules. Defining retention as attendance 'appropriate to need' therefore fully accounts for the spectrum of comorbidity and complex clinical presentations which might interact to increase health disparities.

There are limitations to this research. It is possible that measurement of retention according to the individualized scheduled visits was not appropriate. In the absence of clearly defined timeframes within which to consider attendance as 'on time', the authors defined this within the present study. This is not ideal, and reflects the broader issue of a lack of consistency in measuring and defining retention. In addition, low numbers also inhibited the analyses and the inferences that can be drawn from the data, although this was corrected for statistically as far as possible.

Other important aspects which are missing in the present study include a comparative client self-report of comorbidity presentations. While it was beyond the scope of the present retrospective study to include this, future prospective studies should seek to include this as an important aspect of optimal client-centred care. In addition, in the present study there was no way of knowing whether participants were accessing care elsewhere. Albion is located in metropolitan Sydney, where multiple HIV specialist services exist within a $15 \mathrm{~km}$ radius and there is no centralized system which monitors attendance at services between the private and public sectors. Therefore, it is possible that participants within the AUnotWHO group might have accessed care elsewhere between appointments, thus potentially inflating the estimated rate of poor attendance. This robust data linkage between clinics should be a goal of future research, and the focus of local and State directorates to improve the quality of the data captured with respect to retention.

Another consideration is the association of clinicianassessed comorbidity with clinician-determined schedules. It is possible that the clinician assessment of need did not match the clients', and therefore may account for the discrepancy in retention rates. It is also worth noting that clinician assessment of comorbidity relies upon clients accurately reporting symptoms to their medical practitioners, which may not always occur. For example, there is a likelihood that factors such as problematic CMA use or financial instability may be under-reported to medical doctors, and thus bias their assessment of the same. In addition, it is also possible that those with specific symptoms (e.g. problematic CMA use) may seek specialist services for treatment rather than their HIV clinic; again, this might suggest limited utility of clinician assessment.

A high proportion (97\%) of clients attending this HIV service have an UDVL, which suggests that little of the 
complexity identified relates to HIV specifically, and would therefore be unlikely to translate into a more intensive schedule of visits. While the high proportion of those with a UDVL might seemingly limit the applicability of the findings, it could be argued that retention in care should transcend VL monitoring alone, particularly in the context of the medical and/or psychosocial comorbidity that was present in the sample. This again points to the opportunity to expand reporting requirements to include factors beyond VL alone, such as quality of life.

Further limitations include the use of a relatively new screening tool, which relies upon clinician assessment, and requires further validation. In addition, a dilemma with any retention research is that inclusion in the study relies upon attendance at some point, which automatically fails to capture those who do not attend. The study sample was also relatively young, which might also account for the smaller proportion of clients with higher complexity scores. The present study was also conducted in an urban, well-resourced setting, which limits the generalizability of the findings to similar contexts.

\section{Conclusions}

The present study offers a promising insight to the role of psychosocial and physical health comorbidities in influencing retention, and a signpost to the factors healthcare providers should monitor to optimize care. The significant interaction result in particular warrants further investigation, given its impact on retention in this study. Understanding the comorbid factors which can lead to complex clinical presentations and require an interdisciplinary approach is an important step in improving holistic care for people living with HIV. Reviewing region-specific definitions of retention in care, with a view to standardization where appropriate, is also necessary to ensure existing policies that determine resource allocation are meeting the needs of all people living with HIV, especially the most vulnerable.

\section{Compliance with Ethical Standards}

Conflicts of interest The authors declare they have no conflicts of interest.

Ethical Approval This research did not involve human and/or animal participants; as such no informed consent was sought. Ethics approval for this study was granted by South East Sydney Local Health District (SESLHD; NSW Health) and the University of Technology Sydney (UTS).

\section{References}

1. Grant RW, Ashburner JM, Hong CS, Chang Y, Barry MJ, Atlas SJ. defining patient complexity from the primary care physician's perspective: a cohort study. Ann Intern Med. 2011;155:797-804.

2. Lazarus JV, Safreed-Harmon K, Barton SE, Costagliola D, Dedes N, Del Amo VJ, et al. Beyond viral suppression of HIVthe new quality of life frontier. BMC Med. 2016;14(1):94.

3. Kirby Institute. HIV, viral hepatitis and sexually transmissable infections in Australia: Annual surveillance report 2018. In: Kirby Institute, editor. Sydney, Australia. 2018.

4. British HIV Association. Standards of Care for People Living with HIV 2018. In: British HIV Association, editor. 2018.

5. Centers for Disease Control and Prevention. Understanding the HIV care continuum. In: Centres for Disease Control and Prevention, editor. 2018.

6. World Health Organization. Consolidated Guidelines on Person-Centred HIV Patient Monitoring and Case Surveillance. In: World Health Organization, editor. Geneva, Switzerland. 2017.

7. Singer M, Clair S. Syndemics and public health: reconceptualizing disease in bio-social context. Med Anthropol Q. 2013;17(4):423-41.

8. Mizuno Y, Purcell DW, Knowlton AR, Wilkinson JD, Gourevitch MN, Knight KR. Syndemic vulnerability, sexual and injection risk behaviors, and HIV continuum of care outcomes in HIV-positive injection drug users. AIDS Behav. 2015;19(4):684-93.

9. Wawrzyniak AJ, Rodriguez AE, Falcon AE, Chakrabarti A, Parra A, Park J, et al. Association of individual and systemic barriers to optimal medical care in people living with HIV/ AIDS in Miami-Dade County. J Acquir Immune Defic Syndr. 2015;69(Suppl 1):S63-72.

10. Biello KB, Oldenburg CE, Safren SA, Rosenberger JG, Novak DS, Mayer KH, et al. Multiple syndemic psychosocial factors are associated with reduced engagement in HIV care among a multinational, online sample of HIV-infected MSM in Latin America. AIDS Care. 2016;28(Suppl 1):84-91.

11. Howarth A, Apea V, Michie S, Morris S, Sachikonye M, Mercer C, et al. REACH: a mixed-methods study to investigate the measurement, prediction and improvement of retention and engagement in outpatient HIV care. Health Serv Delivery Res. 2017. https://doi.org/10.3310/hsdr05130.

12. Howarth AR, Burns FM, Apea V, Jose S, Hill T, Delpech VC, et al. Development and application of a new measure of engagement in out-patient HIV care. HIV Med. 2017;18(4):267-74.

13. Bulsara SM, Begley K, Smith DE, Chan DJ, Furner V, Coote $\mathrm{KV}$, et al. The development of an HIV-specific complexity rating scale. Int J STD AIDS. 2019;30(13):1265-74.

14. Mugavero MJ, Davila JA, Nevin CR, Giordano TP. From access to engagement: measuring retention in outpatient HIV clinical care. AIDS Patient Care STDS. 2010;24(10):607-13.

15. Firth D. Bias reduction of maximum likelihood estimates. Biometrika. 1993;80(1):27-38.

16. Tsai AC, Venkataramani AS. Syndemics and health disparities: a methodological note. AIDS Behav. 2016;20(2):423-30.

17. Hosmer DW, Lemeshow S, May S. Applied survival analysis: regression modeling of time-to-event data. New York: WileyInterscience; 2008.

18. Crawford TN. Examining the relationship between multiple comorbidities and retention in HIV medical care: a retrospective analysis. AIDS Care. 2015;27(7):892-9.

19. Bulsara SM, Wainberg ML, Audet CM, Newton-John TRO. Retention in HIV care in Australia: the perspectives of clinicians and clients, and the impact of medical and psychosocial comorbidity. AIDS Patient Care STDs. 2019;33(10):415-24. 
20. Cruwys T, Wakefield JR, Sani F, Dingle GA, Jetten J. Social isolation predicts frequent attendance in primary care. Ann Behav Med. 2018;52(10):817-29.

21. Chua D, Johnson T. Frequent Attenders in Primary Care: A complex set of psychosocial and physiological issues which leads to overburden in GP's diaries and increased strain on health resources. In: Australian Givernment Productivity Commission, editor. QLD, Australia. 2019.

22. Tsai AC, Mendenhall E, Trostle JA, Kawachi I. Co-occurring epidemics, syndemics, and population health. Lancet. 2017;389(10072):978-82.

23. Prestage G, Hammoud M, Jin F, Degenhardt L, Bourne A, Maher L. Mental health, drug use and sexual risk behavior among gay and bisexual men. Int J Drug Policy. 2018;55:169-79.

24. Helleberg M, Engsig FN, Kronborg G, Larsen CS, Pedersen G, Pedersen $\mathrm{C}$, et al. Retention in a public healthcare system with free access to treatment: a Danish nationwide HIV cohort study. AIDS (Lond, Engl). 2012;26(6):741-8.

25. Yehia BR, Fleishman JA, Metlay JP, Korthuis PT, Agwu AL, Berry SA, et al. Comparing different measures of retention in outpatient HIV care. AIDS (Lond, Engl). 2012;26(9):1131-9.

26. Syed IA, Sulaiman SA, Hassali MA, Syed SH, Shan LH, Lee CK. Factors associated with poor CD4 and viral load outcomes in patients with HIV/AIDS. J Med Virol. 2016;88(5):790-7.

Publisher's Note Springer Nature remains neutral with regard to jurisdictional claims in published maps and institutional affiliations. 